The final publications is now available at doi:10.1016/j.jempfin.2015.03.003

\title{
Portfolio optimization for heavy-tailed assets: Extreme Risk Index vs. Markowitz
}

\author{
Georg Mainik * Georgi Mitov ${ }^{\dagger} \quad$ Ludger Rüschendorf ${ }^{\ddagger}$ \\ May 7, 2015
}

\begin{abstract}
Using daily returns of the S\&P 500 stocks from 2001 to 2011, we perform a backtesting study of the portfolio optimization strategy based on the extreme risk index (ERI). This method uses multivariate extreme value theory to minimize the probability of large portfolio losses. With more than 400 stocks to choose from, our study seems to be the first application of extreme value techniques in portfolio management on a large scale. The primary aim of our investigation is the potential of ERI in practice. The performance of this strategy is benchmarked against the minimum variance portfolio and the equally weighted portfolio. These fundamental strategies are important benchmarks for large-scale applications. Our comparison includes annualized portfolio returns, maximal drawdowns, transaction costs, portfolio concentration, and asset diversity in the portfolio. In addition to that we study the impact of an alternative tail index estimator. Our results show that the ERI strategy significantly outperforms both the minimum-variance portfolio and the equally weighted portfolio on assets with heavy tails.
\end{abstract}

Keywords: portfolio optimization; heavy tails; tail risk; extreme risk index; extreme value theory; financial crisis.

*RiskLab, Department of Mathematics, ETH Zurich: Raemistrasse 101, 8092 Zurich, Switzerland, www.georgmainik.com

${ }^{\dagger}$ FinAnalytica Inc. Sofia: 21 Srebarna Str., Floor 5, 1407 Sofia, Bulgaria, georgi.mitov@finanalytica.com

†Department of Mathematical Stochastics, University of Freiburg: Eckerstr. 1, 79104 Freiburg, Germany, ruschen@stochastik.uni-freiburg.de

1

(C)2015, Elsevier. Licensed under the Creative Commons Attribution-NonCommercial-NoDerivatives 4.0 International http: // creativecommons. org/licenses/by-nc-nd/4.0/ 
The final publications is now available at doi:10.1016/j.jempfin.2015.03.003

\section{Introduction}

In this paper we propose and test a portfolio optimization strategy that aims to improve the portfolio return by stabilizing the portfolio value. Minimizing the probability of large drawdowns, this strategy can help to retrieve the portfolio value as good as possible also in times of high risk in the markets. This intended performance is, of course, not a new aim in portfolio management, and it became even more vital since the default of Lehman Brothers in 2008. The following years of financial crisis have demonstrated that the technical progress of financial markets and their globalization have also brought up some new challenges. One of these challenges is the need for diversification strategies that account for strong drawdowns and increasing dependence of asset returns in crisis periods. This has raised the relevance of non-Gaussian models, tail dependence, and quantile based risk measures in portfolio optimization [5, 8, 9, 10, 11, 12, 17, 21, 24, 26, 35, 38, 39, 44.

\section{Developments in theory and practice of portfolio opti- mization}

Since its introduction by Markowitz [36], the mean-variance approach became the industry standard for asset allocation. However, this popularity also brought up several technical issues in practical applications, and there has been a large amount of further development addressing them.

One main direction of related research is dedicated to the impact of $p a-$ rameter uncertainty on the investment performance. The high sensitivity of the estimated mean-variance efficient portfolio to estimation errors in the underlying distribution parameters (expectations and covariances of asset returns) may lead to highly non-robust results. Barry [2] and Chopra and Ziemba [6] show the high sensitivity in particular when estimating the expected returns. Jorion [29, 30, 31] and Jagannathan and Ma [27] find that the pure minimum variance (MV) portfolio may outperform the mean-variance efficient portfolio.

Several approaches addressing the statistical challenge of parameter uncertainty have been suggested in the literature. These include the use of Bayesian and shrinkage estimators, shrinking the portfolios to some predetermined target which depends on combination of prior information with sample data (see, e.g., Jorion [29, 30]). Black and Litterman [3] suggest Bayes estimation of means and covariances. However, their findings on the superiority

(C)2015, Elsevier. Licensed under the Creative Commons Attribution-NonCommercial-NoDerivatives 4.0 International

http: //creativecommons.org/licenses/by-nc-nd/4.0/ 
The final publications is now available at doi:10.1016/j.jempfin.2015.03.003

of the Bayes/Stein procedure are not confirmed in some other studies like Fletcher and Leyffer [16] and Grauer and Hakansson [20]. DeMiguel et al. [9] and DeMiguel and Nogales [8] investigate the potential advantage of robust optimization and shrinkage estimators. The resulting picture is, however, not completely clear, and it turns out that even robustified and optimized procedures in some cases fail to outperform simple heuristic strategies like the equally weighted portfolio.

Concerning robust asset allocation, Tütüncü and Koenig [42] look for robust solutions that have the optimal worst-case performance, whereas Goldfarb and Iyengar [19] choose worst-case estimators in a robust model framework that can be solved by linear programming. Herold and Maurer [22] observe that even these more stable estimation methods only outperform simple strategies when combined with regression models for the expected return.

Another research direction includes several approaches to change the objective function in the optimization problem underlying the investment strategy. One of the issues addressed here is that quantification of risk by variance does not distinguish between gains and losses. Hence, to avoid wrong conclusions for asymmetrically distributed returns, application of pure downside risk measures is advantageous. Young [43] introduces an alternative optimization criterion based on minimum return instead of variance as measure of risk, and proposes a minimax approach. This corresponds to a utility principle with an extreme form of risk aversion on investor's side. Ghaoui et al. [18] propose a worst-case Value-at-Risk and robustified programming approach based on only partial information about the return distributions, assuming that only bounds on the moments are known. Jarrow and Zhao [28] apply lower partial moments as risk measure for downside loss aversion and compare the resulting optimal portfolios with the mean-variance based ones. While both methods perform similarly on normally distributed returns, they can lead to significantly different results on returns with asymmetric, heavy-tailed distributions.

\section{Portfolio optimization based on the Extreme Risk Index (ERI)}

In our paper we follow the basic line of developments on the optimization problem that the investment strategy is derived from. Our reformulation of

(C)2015, Elsevier. Licensed under the Creative Commons Attribution-NonCommercial-NoDerivatives 4.0 International

http: // creativecommons. org/licenses/by-nc-nd/4.0/ 
The final publications is now available at doi:10.1016/j.jempfin.2015.03.003

the objective function in this optimization problem is based on extreme value theory, and it is specifically designed for portfolios with heavy-tailed assets. Extreme value theory is an adequate tool to improve the modelling of return tails.

In contrast to the mean-variance optimization, our approach does not rely on existence of second moments for the return distribution. With increasingly heavy tails, variance and covariance estimators can become unreliable, or even the moment themselves may fail to exist. Thus the mean-variance approach tends to face its limitations especially in crisis periods, when financial returns behave in their most extreme way. Several modifications addressing this issue have been discussed; see, e.g., Rachev et al. [39] for the relevance of this type of heavy-tailed models.

In the present study we apply a novel method based on extreme value theory to a portfolio optimization on real data. This study seems to be the first attempt in extreme-value based portfolio optimization on large scale. Our primary aim is to assess the general potential of extreme-value based methods in portfolio optimization. At this initial stage, we compare a very basic implementation of our extreme-value approach with similarly basic and therefore relatively robust benchmarks. Our benchmarks are given by the minimumvariance portfolio (MV) and the equally weighed portfolio (EW), which invests the $1 / N$ fraction of the total capital in each of $N$ assets. According to our results, the extreme-value based method stays behind its benchmarks on assets with light tails, but outperforms each of them (MV and EW) on assets with moderately heavy or very heavy tails. As discussed above, outperforming these simple methods on large scale is non-trivial even with refined estimation techniques. The advantage of the extreme-value based method is particularly strong in the case of heaviest tails, which the method is designed for.

More specifically, the mathematical basis of our approach is laid out in Mainik and Rüschendorf [35]. Our portfolio is obtained by minimizing the Extreme Risk Index (ERI), which quantifies the impact of heavy, dependent tails of asset returns on the tail of the portfolio return. We apply this strategy and the chosen benchmarks to the daily return data of the S\&P 500 stocks in the period from November 2007 to September 2011. The computation of portfolio weights utilize the data from the six years prior to each trading day. To assess the impact of delays in portfolio rebalancing, we implement rebalancing not only on daily, but also on weekly basis. For the sake of stability, the portfolio estimates for both daily and weekly rebalancing are

(C)2015, Elsevier. Licensed under the Creative Commons Attribution-NonCommercial-NoDerivatives 4.0 International http: //creativecommons.org/licenses/by-nc-nd/4.0/ 
The final publications is now available at doi:10.1016/j.jempfin.2015.03.003

based on daily data. In addition to the portfolio value we also track some other characteristics related to portfolio structure, degree of diversification, and transaction costs.

In the first round of our backtesting experiments we apply ERI optimization to all S\&P 500 stocks with full history in our data set (444 out of 500). In this basic setting the ERI based algorithm slightly outperforms the MV and EW portfolios with respect to annualized returns (6.8\% vs. $5.8 \%$ and $5.3 \%$ for daily rebalancing). All methods significantly outperform the S\&P 500 index, which has the annualized return of $-5.2 \%$.

As next step we subdivide the stocks into three groups according to their tail characteristics. Our results show that ERI optimization is particularly useful for assets with heavy tails. On this asset group it clearly outperforms Markowitz and yields an annualized return of $11.5 \%$ for daily rebalancing. This is impressive compared to the $5.0 \%$ and $5.1 \%$ achieved with the MV and EW strategies, and even more so because the backtesting period includes the recent financial crisis. Tracking the portfolio turnover, we found that the ERI strategy tends to increase the transaction costs. However, the turnover of the ERI optimal portfolio for the group with heavy tails is lower than the turnover of the MV portfolio in the basic experiment without grouping. The performance of the EW portfolio is similar to that of the MV portfolio, especially on assets with heavy-tailed returns.

Our major finding is that the ERI optimization significantly outperforms MV and EW portfolios for assets with very heavy tails. Furthermore, the structure of the ERI optimal portfolio is very different from its peers, especially in the basic case with portfolio selection from all 444 assets considered. The ERI based portfolio is build from fewer assets, but nevertheless it shows better diversification as measured by principal component analysis. The overall picture for weekly rebalancing is similar. These results suggest that ERI optimization can be a useful alternative for portfolio selection in risky asset classes. In some sense, this strategy seems to earn the reward that the economic theory promises for the higher risk of heavier tails.

A remarkable detail in this study is that none of the three compared methods (ERI, MV, EW) looks at expected returns. Nevertheless, each of them significantly outperforms the S\&P 500 index, and the annualized return of the ERI strategy on heavy-tailed assets is surprisingly high if we keep in mind that the data we used includes the financial crisis of 2008 and 2009. The risk-orientated nature of the ERI strategy suggests that this result is due to improved detection and handling of risk in the portfolio.

(C)2015, Elsevier. Licensed under the Creative Commons Attribution-NonCommercial-NoDerivatives 4.0 International

http: //creativecommons.org/licenses/by-nc-nd/4.0/ 
The final publications is now available at doi:10.1016/j.jempfin.2015.03.003

Further improvement of the ERI-based portfolio optimization by incorporating expected returns is analogous to the mean-variance setting. It can be done by adjusting the ERI-based optimization problem by a linear constraint that reflects some target return. Theoretically, this should improve the performance of the ERI strategy even further. However, practical implementation of this extension faces same statistical challenges as for the Markowitz strategy with a target return. The literature discussed above suggests that outperforming the purely risk-orientated version of the ERI strategy would be non-trivial.

The paper is organized as follows. The alternative portfolio optimization algorithm and its technical backgrounds are introduced in Section 2 In Section 3 we give an outline of the data used in the backtesting study, define the estimator for the optimal portfolio, and introduce all additional portfolio characteristics to be tracked. Detailed results of the backtesting experiments are presented and discussed in Section 4. Conclusions are given in Section 5.

\section{Theoretical backgrounds}

\subsection{Asset and portfolio losses}

Let $S_{i}(t)$ denote prices of assets $S_{i}, i=1, \ldots, N$, at times $t=0,1, \ldots, T$. Focusing on the downside risk, let $X_{i}(t)$ denote the logarithmic losses of the assets $S_{i}$,

$$
X_{i}(t):=-\log \left(\frac{S_{i}(t)}{S_{i}(t-1)}\right)=\log S_{i}(t-1)-\log S_{i}(t),
$$

and let $\widetilde{X}_{i}(t)$ denote the corresponding relative losses:

$$
\widetilde{X}_{i}(t):=\frac{S_{i}(t-1)-S_{i}(t)}{S_{i}(t)}=\frac{S_{i}(t-1)}{S_{i}(t)}-1 .
$$

For daily stock returns, $X_{i}$ and $\widetilde{X}_{i}$ are almost identical because $\widetilde{X}_{i}$ is the first-order Taylor approximation to the logarithmic loss $X_{i}$.

This approximation also extends to asset portfolios. Consider an investment strategy (static or one-period) diversifying a unit capital over the assets $S_{1}, \ldots, S_{N}$. It can be represented by a vector $w$ of portfolio weights, $w \in$ $H_{1}:=\left\{x \in \mathbb{R}^{N}: \sum_{i=1}^{N} x_{i}=1\right\}$. Excluding short positions, the portfolio set 
The final publications is now available at doi:10.1016/j.jempfin.2015.03.003

can be restricted to the unit simplex $\Delta^{N}:=\left\{w \in[0,1]^{N}: \sum_{i=1}^{N} w_{i}=1\right\}$. This is the portfolio set we will work with from now on. Each component $w_{i} \geq 0$ corresponds to the fraction of the total capital invested in $S_{i}$, and the relative portfolio loss is equal to the scalar product $w^{T} \widetilde{X}(t):=\sum_{i=1}^{N} w_{i} \widetilde{X}_{i}(t)$ of the portfolio vector $w$ and the relative loss vector $\widetilde{X}(t)=\left(\widetilde{X}_{1}(t), \ldots, \widetilde{X}_{N}(t)\right)$ :

$$
\sum_{i=1}^{N} \frac{w_{i}}{S_{i}(t-1)}\left(S_{i}(t-1)-S_{i}(t)\right)=w^{T} \widetilde{X}(t) .
$$

Thus the scalar product $w^{T} X(t)$ for the logarithmic loss vector $X(t):=$ $\left(X_{1}(t), \ldots, X_{N}(t)\right)$ is the first-order Taylor approximation to $w^{T} \widetilde{X}$. This kind of approximation is also relevant to the Markowitz approach, which is typically applied to logarithmic returns.

\subsection{Multivariate regular variation}

To define the Extreme Risk Index (ERI) of the random vector $X(t)$, we recollect the notion of multivariate regular variation (MRV). A random vector $X=\left(X_{1}, \ldots, X_{N}\right)$ is MRV if the joint distribution of its polar coordinates $R:=\|X\|_{1}:=\sum_{i=1}^{N}\left|X_{i}\right|$ and $Z:=\|X\|_{1}^{-1} X$ satisfies

$$
\mathcal{B}\left(\left(r^{-1} R, Z\right) \mid R>r\right) \stackrel{w}{\rightarrow} \rho_{\alpha} \otimes \Psi, \quad r \rightarrow \infty,
$$

where $\Psi$ is a probability measure on the 1-norm unit sphere $\mathbb{S}_{1}^{N}$ and $\rho_{\alpha}$ is the Pareto distribution: $\rho_{\alpha}(s, \infty)=s^{-\alpha}, s \geq 1$. The symbol $\stackrel{w}{\rightarrow}$ in $(2.3)$ represents the weak convergence of probability measures, and the symbol $\otimes$ refers to the direct product of probability measures. The intuitive meaning of (2.3) is that, conditioned on $R>r$ for a sufficiently large $r$, the random variable $r^{-1} R$ is approximately $\operatorname{Pareto}(\alpha)$ distributed and independent of $Z$, which is approximately $\Psi$-distributed.

Besides (2.3), there are several other equivalent definitions of MRV; for more details we refer to Resnick [40]. The parameter $\alpha>0$ is called tail index. It separates finite moments of $R$ from infinite ones in the sense that $E R^{\beta}<\infty$ for $\beta<\alpha$ and $E R^{\beta}=\infty$ for $\beta>\alpha$. In the non-degenerate case, the same moment explosion occurs for all components $X_{i}$ of the random vector $X$. The measure $\Psi$ is called spectral (or angular) measure of $X$ and describes the asymptotic distribution of excess directions for the random vector $X$.

(C)2015, Elsevier. Licensed under the Creative Commons Attribution-NonCommercial-NoDerivatives 4.0 International http: //creativecommons.org/licenses/by-nc-nd/4.0/ 
The final publications is now available at doi:10.1016/j.jempfin.2015.03.003

Intuitively speaking, MRV means that the radius $R$ has a polynomial tail and is asymptotically (i.e., for large $R$ ) independent of the angular part $Z$. Moreover, if a measurable set $A \subset \mathbb{R}^{N}$ is sufficiently far away from the origin, i.e., if $\|x\|_{1} \geq t$ for all $x \in A$ with some large $t$, then

$$
P(X \in s A) \simeq s^{-\alpha} P(X \in A)
$$

for $s \geq 1$ and $s A:=\{s x: x \in A\}$. The scaling property (2.4) allows to extrapolate from large losses to extremely large ones, which even may be beyond the range of the observed data. Approximations of this kind are the key idea of the Extreme Value Theory (cf. Embrechts et al. [15]).

Many popular models are MRV. In particular, this is the case for multivariate $t$ and multivariate $\alpha$-stable distributions (cf. Araujo and Giné [1], Hult and Lindskog [25]). In the latter case, the stability index $\alpha$ is also the tail index, and the spectral measure characterizing the multivariate stability property is a constant multiple of $\Psi$ from (2.3). In all these models, the components $X_{i}$ are tail equivalent in the sense that $P\left(X_{i}>r\right) / P\left(X_{j}>\right.$ $r) \rightarrow c_{i, j}>0$ as $r \rightarrow \infty$ for all $i, j \in\{1, \ldots, N\}$. This is equivalent to the following non-degeneracy condition for the angular measure $\Psi$ :

$$
\Psi\left\{x \in \mathbb{S}_{1}^{N}: x_{i}=0\right\}<1
$$

for $i=1, \ldots, N$.

It should be noted that the MRV assumption (2.3) is of asymptotic nature and that it is also quite restrictive. MRV models are often criticized for excluding even slightly different tail indices $\alpha_{i}$ for the components $X_{i}$. However, this criticism also affects the multivariate $t$ and multivariate $\alpha$ stable models, which are widely accepted in practice despite the resulting restriction to equal $\alpha_{i}$. It is indeed true that, estimating the tail index $\alpha_{i}$ for each component $X_{i}$ separately, one would hardly ever obtain identical values for different $i$. But on the other hand, the confidence intervals for $\alpha_{i}$ often overlap, so that a MRV model may be close enough to reality and provide a useful result.

The major reason why MRV models can be useful in practice is that the practical questions are non-asymptotic. In fact, it is not the restrictive asymptotic relation (2.3) that matters, but the scaling property (2.4). If (2.4) is sufficiently close to reality in the range that is relevant to the application, the eventual violation of (2.3) further out in the tails does not influence the result too much.

(C)2015, Elsevier. Licensed under the Creative Commons Attribution-NonCommercial-NoDerivatives 4.0 International http: // creativecommons. org/licenses/by-nc-nd/4.0/ 
The final publications is now available at doi:10.1016/j.jempfin.2015.03.003

Practical applications often involve heuristics of this kind. In particular, if $S_{i}$ are stock prices and hence non-negative, then the relative losses $\widetilde{X}_{i}$ are bounded by 1 . Going sufficiently far out into the tail, one must observe quite different behaviours for the relative portfolio loss $w^{T} \widetilde{X}$ and the logarithmic approximation $w^{T} X$. However, with typical daily return values in the low percentage area and values around $10 \%$ occurring only in crisis times, relative asset losses do exhibit polynomial scaling of the type

$$
\frac{P\left(\widetilde{X}_{i}>r s\right)}{P\left(\widetilde{X}_{i}>r\right)} \simeq s^{-\alpha} .
$$

This is illustrated in Figure 2.1, which shows QQ-plots of logarithmic S\&P 500 returns (same observation period as in our backtesting study) versus the normal and the Student- $t$ (3) distribution. The normal distribution is light-tailed, whereas the $t(3)$ distribution satisfies (2.5) with $\alpha=3$ (more generally, a $t$ distribution with $\nu$ degrees of freedom satisfies (2.5) with $\alpha=\nu$ ). The dashed, red lines mark the $0.4 \%, 10 \%, 90 \%$, and $99.6 \%$ quantiles of the distributions on the $x$ axes (normal or $t(3)$ ). The area between the $0.4 \%$ and $10 \%$ quantiles corresponds to the worst returns observed every 2 weeks (10 business days) or once a year (about 250 business days). The area between the $90 \%$ and $99.6 \%$ quantiles corresponds to the best returns observed every 2 weeks or once a year. This is the application range mentioned above. A good distributional fit makes the QQ-plot linear in this range. Figure 2.1 demonstrates clearly that the normal distribution gives a poor fit to the S\&P 500 return data, whereas the heavy-tailed $t$ distribution fits much better. This picture depends neither on whether one takes the index or single stocks, nor on the observation period. Figure 2.1 uses the same observation range as our backtesting study, but even shifting the observation window 10 or 20 years back into the past gives astonishingly similar results.

Hence we are lucky to remain in the area where $X$ and $\widetilde{X}$ can be treated as if they both were MRV, and the approximation $w^{T} \widetilde{X} \simeq w^{T} X$ works reasonably well. Thus, even though the scaling property $(2.5)$ eventually breaks down if $r s$ gets too close to 1 , it has some useful consequences in the application range. This is confirmed by our backtesting results.

(C)2015, Elsevier. Licensed under the Creative Commons Attribution-NonCommercial-NoDerivatives 4.0 International http: //creativecommons.org/licenses/by-nc-nd/4.0/ 
The final publications is now available at doi:10.1016/j.jempfin.2015.03.003
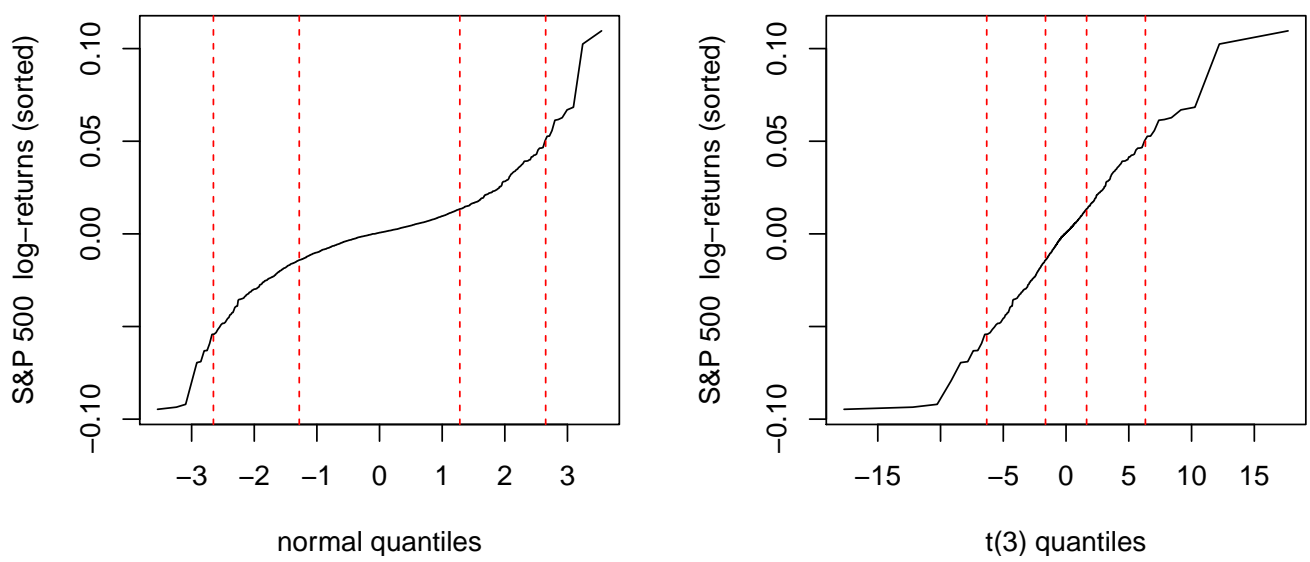

Figure 2.1 QQ-plots for the logarithmic returns of the S\&P 500 index vs. normal and $t(3)$ distribution. The dashed, red lines mark the $0.4 \%, 10 \%, 90 \%$, and $99.6 \%$ quantiles.

\subsection{Portfolio optimization via Extreme Risk Index}

The MRV assumption (2.3) implies that

$$
\lim _{r \rightarrow \infty} \frac{P\left(w^{T} X>r\right)}{P\left(\|X\|_{1}>r\right)}=\gamma_{w}:=\int_{\mathbb{S}_{1}^{N}} \max \left(0, w^{T} z\right)^{\alpha} d \Psi(z)
$$

(cf. 35 and 33, Lemma 2.2). This implies that for any portfolio vectors $v, w \in \Delta^{N}$ and large $r>0$

$$
\frac{P\left(v^{T} X>r\right)}{P\left(w^{T} X>r\right)} \simeq \frac{\gamma_{v}}{\gamma_{w}} .
$$

Moreover, for $\lambda \leq 1$ close to 1 one obtains that

$$
\frac{\operatorname{VaR}_{\lambda}\left(v^{T} X\right)}{\operatorname{VaR}_{\lambda}\left(w^{T} X\right)} \simeq\left(\frac{\gamma_{v}}{\gamma_{w}}\right)^{1 / \alpha}
$$

(cf. 35 and 34, Corollary 2.3). Here and in what follows we define the Valueat-Risk $\mathrm{VaR}_{\lambda}$ of a random loss $X$ at confidence level $\lambda$ as the $\lambda$-quantile of $X$ :

$$
\operatorname{VaR}_{\lambda}(X):=\inf \{x \in \mathbb{R}: P(X \leq x) \geq \lambda\} .
$$

Roughly speaking, $\operatorname{VaR}_{\lambda}(X)$ is the smallest $x$ such that $X \leq x$ holds with probability $\lambda$. Typical values of $\lambda$ are $95 \%, 99 \%$, and $99.5 \%$. 
The final publications is now available at doi:10.1016/j.jempfin.2015.03.003

Motivated by (2.7) and (2.8), the functional $\gamma_{w}=\gamma_{w}(\Psi, \alpha)$ is called $E x$ treme Risk Index (ERI). Minimizing the function $w \mapsto \gamma_{w}$, one obtains a portfolio that minimizes the loss for large $\|X\|$, i.e., in case of crisis events. In precise mathematical terms, one minimizes $\operatorname{VaR}_{\lambda}\left(w^{T} X\right)$ for $\lambda \rightarrow 1$. The practical meaning of this procedure is the utilization of the scaling property (2.4) to obtain a portfolio that minimizes the downside risk during a market crash. This approximate result is not perfect, but it can be a step into the right direction.

Based on the integral representation (2.6), the following portfolio optimization approach was proposed in Mainik and Rüschendorf [35]:

- Estimate $\gamma_{w}$ by plugging appropriate estimates for $\alpha$ and $\Psi$ into (2.6);

- Estimate the optimal portfolio by minimizing the resulting estimator $\widehat{\gamma}_{w}$ with respect to $w$.

The general properties of the optimization problem are discussed in Mainik and Rüschendorf [35], Mainik [32], and Mainik and Embrechts [34]. In particular, it is known that the function $w \mapsto \widehat{\gamma}_{w}$ is convex for $\alpha>1$. Thus, given that the expectations of $X_{i}$ are finite, a typical optimal portfolio would diversify over multiple assets. The consistency of the plug-in estimator $\widehat{\gamma}_{w}$ and of the resulting estimated optimal portfolio $w^{*}$ in a strict theoretical sense is studied in Mainik [32, 33], Mainik and Rüschendorf [35].

\section{Outline of the backtesting study}

\subsection{The data}

The contribution of the present paper is a backtesting study of the ERI based portfolio optimization approach on real market data. Our data set comprises all constituents of the S\&P 500 market index that have a full history for the period of 10 years back from 19-Oct-2011. These are 444 stocks out of 500. For each date of the backtest period 19-Oct-2007 to 19-Oct-2011 the estimation of the optimal portfolio is based on the 1500 foregoing observations - approximately 6 years of history - for all stocks back in time. For example, the optimal portfolio for 19-Oct-2007 is estimated from the stock price data for the period (19-Oct-2001 to 18-Oct-2007).

Our computations are based on the logarithmic losses $X_{i}(t)$ as defined in (2.1). As already mentioned above, we exclude short positions. This

(C)2015, Elsevier. Licensed under the Creative Commons Attribution-NonCommercial-NoDerivatives 4.0 International

http: //creativecommons.org/licenses/by-nc-nd/4.0/ 
The final publications is now available at doi:10.1016/j.jempfin.2015.03.003

basic framework is most natural for the comparison of portfolio strategies. The asset index $i$ varies between 1 and $N=444$, and the time index $t$ takes values between 1 and $T=2509$ (1500 days history +1009 days in the backtest period). To estimate $\alpha$ and $\Psi$, we transform the (logarithmic) loss vectors $X(t)$ into polar coordinates

$$
(R(t), Z(t))=\left(\|X(t)\|_{1},\|X(t)\|_{1}^{-1} X(t)\right), \quad t=1, \ldots, T .
$$

\subsection{The estimators and the algorithms}

We estimate $\alpha$ by applying the Hill estimator to the radial parts $R(t)$ :

$$
\widehat{\alpha}=\frac{k}{\sum_{j=1}^{k} \log \left(R_{(j), t} / R_{(k+1), t}\right)}
$$

where $t>1500$ and $R_{(1), t} \geq \ldots \geq R_{(1500), t}$ is the descending order statistic of the radial parts $R(t-1500), \ldots, R(t-1)$ and $k=150$. That is, out of the 1500 data points in the historical observation window $t-1500, \ldots, t-1$ we use the 10\% with largest radial parts. Going back to Hill [23, the Hill estimator is the most prototypical approach for the estimation of the tail index $\alpha$. The choice of $k$ determines which observations are assumed to describe the tail behaviour. Another important criterion for the choice of $k$ is the trade-off between the bias, which typically increases for large $k$, and the variance of the estimator, which increases for small $k$. In addition to the static 10\%-rule we also consider the adaptive approach proposed in Nguyen and Samorodnitsky [37]. See Daníelsson et al. [7], Drees and Kaufmann [14], Resnick and Stărică [41] for further related methods.

As proposed in Mainik and Rüschendorf [35], we estimate $\Psi$ by the empirical measure of the angular parts from observations with largest radial parts. More specifically, we use the same $10 \%$ data points (the so-called tail fraction) in the moving observation window that were used to obtain $\widehat{\alpha}$. The resulting estimator $\gamma_{w}$ is

$$
\widehat{\gamma}_{w}(t)=\frac{1}{k} \sum_{j=1}^{k} \max \left(0, w^{T} Z\left(i_{j, t}\right)\right)^{\widehat{\alpha}},
$$

where $i_{j, t}$ is the sample index of the order statistic $R_{(j), t}$ in the full data set:

$$
R_{(j), t}=R\left(i_{j, t}\right), \quad j=1, \ldots, 1500, \quad t=1501, \ldots, T .
$$


The final publications is now available at doi:10.1016/j.jempfin.2015.03.003

The resulting estimate of the optimal portfolio $w^{*}(t)$ on the trading day $t$ is the portfolio vector $w \in \Delta^{N}$ that minimizes $\widehat{\gamma}_{w}(t)$ :

$$
\widehat{\gamma}_{w^{*}(t)}=\min _{w \in \Delta^{N}} \widehat{\gamma}_{w}(t) .
$$

Finally, the estimated optimal portfolio $w^{*}(t)$ is used to compose the portfolio for the trading day $t$. The resulting (relative) portfolio return is calculated by substituting $w^{*}(t)$ in 2.2 .

The procedure outlined above is repeated for all trading days $t>1500$. For instance, the optimal portfolio for 22-Oct-2007 is based on the observation window from 29-Oct-2001 to 21-Oct-2007, whereas for 23-Oct-2007 we use the observation window from 30-Oct-2001 to 22-Oct-2007, and so on.

The benchmarks for this portfolio optimization algorithm are given by the equally weighted (EW) portfolio assigning the weight of $1 / N=1 / 444$ to each asset and the the minimum variance (MV) portfolio. Analogously to the ERI optimal portfolio, the MV portfolio is calculated from logarithmic asset returns, with the same moving observation window of 1500 points and empirical estimators for the covariance matrix. Similarly to the ERI approach, our implementation of the Markowitz approach chooses the portfolio with minimal risk, i.e. with minimal variance. That is, given an estimator $\widehat{C}$ of the covariance matrix of the asset returns $S_{1}, \ldots, S_{N}$, the estimated minimum variance portfolio $w_{\mathrm{MV}}$ is obtained by minimizing the function

$$
w \mapsto w^{T} \widehat{C} w
$$

for $w \in \Delta^{N}$. That is, we do not include an additional linear constraint

$$
w^{T} \widehat{\mu}=\bar{\mu}
$$

with an estimator $\widehat{\mu}$ of the daily return and a target return $\bar{\mu}>0$.

There are two reasons for this choice. On the one hand, ERI minimization is also a pure risk minimization procedure, so that ignoring estimates of the expected returns in the Markowitz benchmark increases the fairness of competition. Furthermore, since the ERI approach only changes the quantification of risk and does not yet change the view on gains, it is natural to study its effect in a purely risk orientated setting. Endowment of the ERI approach with a target return is straightforward. Analogously to the Markowitz approach, it suffices to add the linear constraint (3.3) to the optimization problem $(3.2)$. Attribution-NonCommercial-NoDerivatives 4.0 International http: //creativecommons.org/licenses/by-nc-nd/4.0/ 
The final publications is now available at doi:10.1016/j.jempfin.2015.03.003

On the other hand, computation of a Markowitz efficient portfolio with target return constraint (3.3) would require estimation of expected asset returns and bring in all the technical issues discussed in Section 1. The same issues must appear in an extended ERI application that includes (3.3). In practice, these technical issues can dominate the theoretical performance improvement associated with a target return.

\section{Empirical results}

\subsection{Basic setting for entire set of stocks}

We start with the most crude application of the ERI minimization strategy, estimating the tail index from the radial parts of the random vector $\left(X_{1}, \ldots\right.$, $X_{444}$ ) of all stock retrains involved in our study. The resulting estimate $\widehat{\alpha}=\widehat{\alpha}(t)$ varies in time, but it is applied to all $N=444$ stocks as if their joint distribution were MRV. This is a very courageous assumption, but even in this case we see some useful results. A first impression of these results is given in Figure 4.1, where the value of the ERI optimal portfolio is compared to the performance of its peers (MV and EW) and to the S\&P 500 index. The graphic suggests that the value of the ERI based portfolio is more stable during market crashes. On the other hand, the MV portfolio seems to catch up again during recovery periods. Markowitz approach also tries to assess potential gains. The cumulative returns achieved with ERI, MV, and EW, are similar in this setting, but still with some advantage for the ERI based portfolio. Thus it seems that the ERI strategy - even in its crudest implementation - has a potential to stabilize the portfolio value in crises. The overall performance of the EW portfolio is very similar to that of the MV portfolio, but with lower Sharpe ratio and higher drawdowns. Thus it suffices to consider the MV benchmark in the present setting.

All actively traded strategies benchmarks (ERI, MV, EW) clearly outperform the S\&P 500 index. The real dimension of this advantage is, however, not obvious, because for simplicity of implementation we apply ERI, MV, and EW only to the 444 stocks that remain in the S\&P 500 through the whole observation period. Since this information about future developments is not available in reality, there may be a survivor bias increasing the performance of the three actively traded benchmarks. However, the resulting comparison between ERI, MV, and EW should be fair because each of them is applied

(C)2015, Elsevier. Licensed under the Creative Commons Attribution-NonCommercial-NoDerivatives 4.0 International

http: //creativecommons.org/licenses/by-nc-nd/4.0/ 
The final publications is now available at doi:10.1016/j.jempfin.2015.03.003

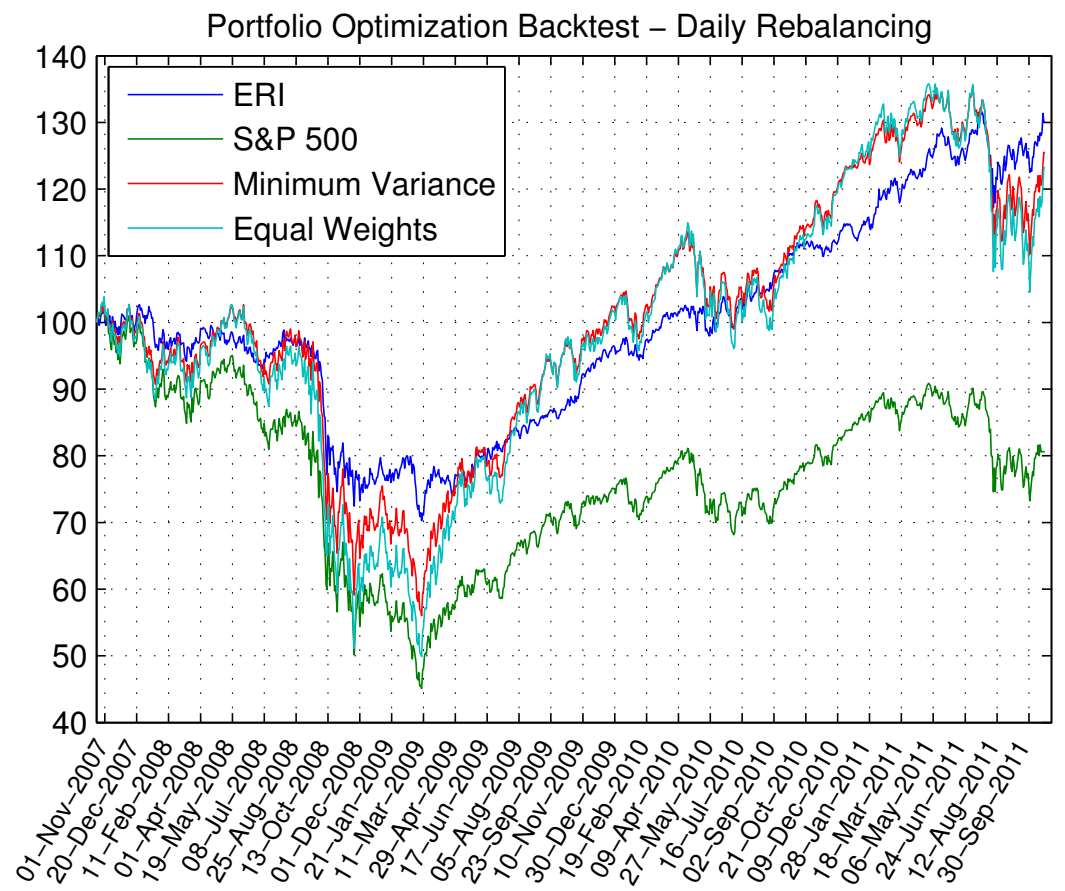

Figure 4.1 Portfolio optimization backtest for the ERI minimization strategy under the assumption that all all stock returns have the same tail index $\alpha$. The resulting portfolio value of the ERI strategy and its peers (MV, EW, and S\&P 500) is scaled to 100 for the first date of the backtest period.

to the 444 survivor stocks.

Further characteristics of the basic ERI approach compared to its peers are shown in Table 4.1. The numbers show that the ERI strategy indeed outperforms MV and EW portfolios in many respects. In particular, the ERI optimal portfolio gives higher cumulative returns and a higher Sharpe ratio, whereas the maximal drawdown is lower than with the MV strategy. An extension of the Sharpe ratio based on the Expected Shortfall (ES) is the STARR ratio (cf. Rachev et al. [39]):

$$
\operatorname{STARR}_{\lambda}(Z):=\frac{\mathrm{E}\left(Z-r_{f}\right)}{\operatorname{ES}_{\lambda}\left(Z-r_{f}\right)}
$$

where $r_{f}$ is the risk-free interest rate and $\lambda$ is a confidence level close to 1 . The backtested STARR is also higher for the ERI strategy than for the MV approach. The computation of the Sharpe and STARR ratios is based on empirical estimators for the expectation and for the Expected Shortfall. In 
The final publications is now available at doi:10.1016/j.jempfin.2015.03.003

\begin{tabular}{lcccc}
\hline \hline & ERI & MV & EW & S\&P 500 \\
\hline \hline CR (Cumulative Return) & $30.07 \%$ & $25.48 \%$ & $23.24 \%$ & $-19.38 \%$ \\
\hline AR (Annualized Return) & $6.76 \%$ & $5.81 \%$ & $5.34 \%$ & $-5.22 \%$ \\
\hline AS (Annualized Sharpe) & 0.4715 & 0.3469 & 0.3229 & -0.0462 \\
\hline AST (Annualized STARR $\left.{ }_{0.95}\right)$ & 0.1926 & 0.1410 & 0.1318 & -0.0187 \\
\hline MD (Max Drawdown) & $46.61 \%$ & $58.61 \%$ & $63.27 \%$ & $56.34 \%$ \\
\hline AC (Average Concentration Coefficient) & 8.69 & 127.22 & 444 & $\mathrm{~N} / \mathrm{A}$ \\
\hline AT (Average Turnover) & 0.0400 & 0.0272 & 0.01 & $\mathrm{~N} / \mathrm{A}$ \\
\hline PCA (First PCA factor Explained Variance) & $31.32 \%$ & $35.48 \%$ & $38.80 \%$ & $\mathrm{~N} / \mathrm{A}$ \\
\hline \hline
\end{tabular}

Table 4.1 Backtest statistics for the ERI minimization strategy in the basic setting (applied to all stocks at once) vs. Minimum Variance (MV), Equally weighted portfolio (EW), and S\&P 500.

particular, the estimate of $\mathrm{ES}_{0.95}$ over the backtesting period of 1009 days is based on 51 largest observations of the portfolio loss. Since a risk-free rate on a daily scale is both difficult to determine and negligibly small, we set $r_{f}=0$. The annualized Sharpe and STARR ratios reported in Table 4.1 and all other tables across the paper are obtained from daily ratios by multiplying them with the factor $\sqrt{252}$. This heuristic approach is based on the assumption hat the calendar year has $T=252$ business days and the returns scale over time with factor $T$, whereas the yearly volatility and Expected Shortfall scale with factor $\sqrt{T}$. The resulting annualized values are very rough approximations, but with 10 years of data, more reliable estimation of yearly returns, volatilities, and Expected Shortfall is not feasible.

To measure the portfolio stock concentration, we compute the Concentration Coefficient (CC). It is defined as

$$
\mathrm{CC}(t):=\left(\sum_{i=1}^{n} w_{i}^{2}(t)\right)^{-1}
$$

where $w_{i}(t)$ is the relative weight of the asset $i$ in the investment portfolio at time $t$. Conceptually, this approach is well known in measures of industrial concentration, where it is called as the Herfindahl-Hirschman index (HHI). Brandes Institute introduced the concentration coefficient by inverting the

16

(C)2015, Elsevier. Licensed under the Creative Commons Attribution-NonCommercial-NoDerivatives 4.0 International http: //creativecommons.org/licenses/by-nc-nd/4.0/ 
The final publications is now available at doi:10.1016/j.jempfin.2015.03.003

HHI.

The CC of an equally weighted portfolio is identical with the number of assets. As the portfolio becomes concentrated on fewer assets, the CC decreases proportionally. The numbers in Table 4.1 indicate that the ERI strategy is quite selective, whereas the number of stocks in the MV portfolio is on the same scale with the total number of assets.

To assess the level of diversification provided by each optimization algorithm, we performed Principal Component Analysis (PCA) over the returns of all stocks relevant to the corresponding portfolios. We defined relevance via portfolio weights assigned by the algorithms and restricted PCA to the stocks with portfolio weights higher than $0.01 \%$. Then we estimated the portion of the sample variance explained by the first PCA factor and averaged these daily estimates over the backtesting period. The lower the average portion of sample variance explained by the first PCA factor, the higher is the portfolio diversification. The numbers in Table 4.1 are quite surprising: despite the significantly higher concentration, the diversification level of the ERI based portfolio is higher than that of the MV strategy.

The only performance characteristic where ERI stays behind MV is the portfolio turnover, which is a proxy to the transaction costs of a strategy. We use a definition of portfolio turnover that is based on the absolute values of the rebalancing trades:

$$
\tau(t):=\sum_{i=1}^{n}\left|w_{i}(t)-w_{i}\left(t_{-}\right)\right|
$$

where $w_{i}(t)$ is the (relative) portfolio weight of the asset $i$ after rebalancing (according to the optimization strategy) at time $t$, and $w_{i}\left(t_{-}\right)$is the portfolio weight of the asset $i$ before rebalancing at time $t$, i.e., at the end of the trading period $t-1$. Averages of $\tau(t)$ over all $t$ in the backtesting period are given in Table 4.1. The average turnover of the ERI optimal portfolio (0.0400) is higher than that of the minimum variance portfolio (0.0272).

Some technical details. For the calculation of the portfolio value we use relative returns and do not expect much difference when using logarithmic approximations. In the calculation of STARR and Sharpe ratio we do not use risk free rates since these are very small on a daily basis and thus have little influence on the the ratio calculations. For the estimation of ES in STARR we use the average of all sample values smaller than the $95 \% \mathrm{VaR}$ of the sample. Our backtest period is of length 1009 and thus the ES estimate is based on $n=51$ observations.

17

(c)2015, Elsevier. Licensed under the Creative Commons Attribution-NonCommercial-NoDerivatives 4.0 International

http: // creativecommons. org/licenses/by-nc-nd/4.0/ 
The final publications is now available at doi:10.1016/j.jempfin.2015.03.003

\subsection{Grouping the stocks with similar $\alpha$}

In the previous section we treated all stocks as if their (logarithmic) returns $X_{i}$ had the same tail index $\alpha$. This simplification can influence the quantitative and qualitative results. To obtain a better insight, we divide the stocks into three different groups with respect to their individual $\alpha$ and compare the performance of the portfolio optimization strategies on each of these groups. Figure 4.2 shows the histogram of the estimates of the tail index $\alpha$ for different stocks on the first day of the backtesting period $(t=1501)$.

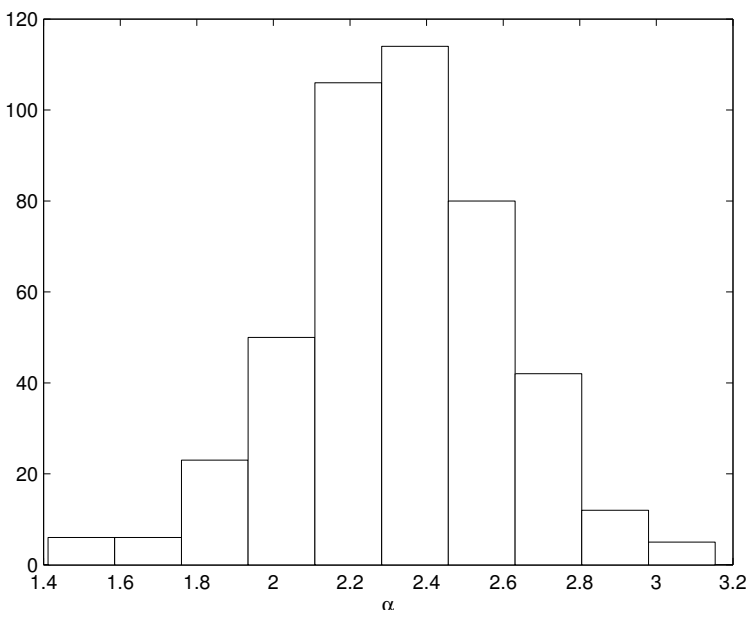

Figure 4.2 Estimated values of the tail index $\alpha$ for different stocks on the first day of the backtesting period

We consider the following groups:

1. all stocks with $\alpha \leq 2.2$

2. all stocks with $\alpha \in(2.2,2.6)$

3. all stocks with $\alpha \geq 2.6$

The first group contains 134, the second 243, and the third one 67 stocks. These groups remained static during the backtesting period. That is, the estimated $\alpha$ on the first day of the backtesting period determines in which group each stock is placed. 
The final publications is now available at doi:10.1016/j.jempfin.2015.03.003

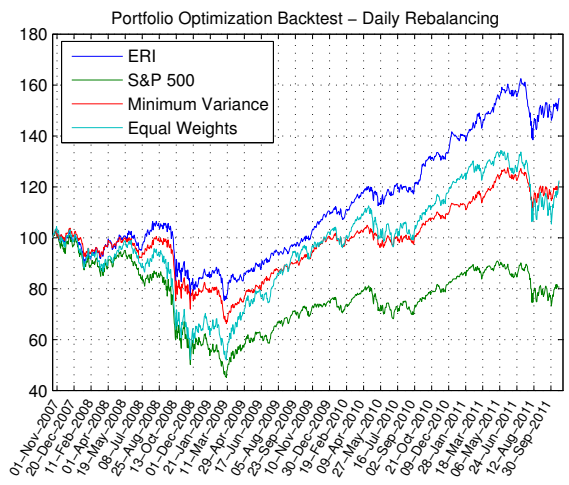

\begin{tabular}{lcccc}
\hline \hline & ERI & MV & EW & S\&P 500 \\
\hline CR & $54.70 \%$ & $21.58 \%$ & $22.30 \%$ & $-19.38 \%$ \\
\hline AR & $11.48 \%$ & $4.99 \%$ & $5.14 \%$ & $-5.22 \%$ \\
\hline AS & 0.6623 & 0.3546 & 0.3182 & -0.0462 \\
\hline AST & 0.2695 & 0.1430 & 0.1299 & -0.0187 \\
\hline MD & $53.67 \%$ & $48.03 \%$ & $61.32 \%$ & $56.34 \%$ \\
\hline AC & 7.4499 & 10.9712 & 134 & N/A \\
\hline AT & 0.0269 & 0.0154 & 0.01 & N/A \\
\hline PCA & $35.02 \%$ & $33.33 \%$ & $35.17 \%$ & N/A \\
\hline \hline
\end{tabular}

Table 4.2 Backtest statistics. Stocks with $\alpha \leq 2.2$

Figure 4.3 Portfolio optimization backtest. Stocks with $\alpha \leq 2.2$

\section{Selection from the set of stocks with $\alpha \leq 2.2$}

The backtesting results on stocks with tail index $\alpha \leq 2.2$ are summarized in Figure 4.3 and Table 4.2 . In this case ERI minimization clearly outperforms its peers and yields an impressive annualized return of $11.48 \%$. This is more than the double of roughly $5 \%$ achieved with the MV or with the EW portfolio. The overall performance of the EW portfolio is again similar to that of the MV portfolio, but with greater drawdowns. Thus it suffices to consider the MV benchmark in this case.

The Sharpe and STARR ratios of the ERI strategy are also clearly higher than with MV. The concentration of both portfolios is on the same scale, but still a bit higher for the ERI based one. Similarly to the basic backtesting set-up on all S\&P 500 stocks, the ERI strategy produces a higher portfolio turnover (0.0269 vs. 0.0154 with MV). However, both values are lower than the average turnover of the MV portfolio in the basic setting (0.0272).

These results suggest that the ERI strategy is particularly useful for optimizing portfolios of stocks with heavy tails, in our case of 134 out of 444 stocks. This is to be expected since the ERI methodology was developed for heavy-tailed MRV models. Beyond that, there is also a statistical reason for the inferior performance of the MV approach in the present setting. Estimation of covariances becomes increasingly difficult for heavier tails, and for $\alpha<2$ the covariances (and hence correlations) do not even exist. Thus empirical covariances used in the Markowitz approach can push the investor into the wrong direction. 
The final publications is now available at doi:10.1016/j.jempfin.2015.03.003

Selection from the set of stocks with $\alpha \in(2.2,2.6)$

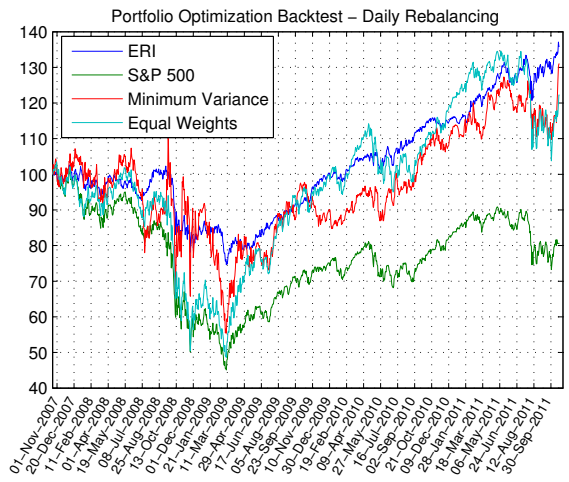

\begin{tabular}{lcccc}
\hline \hline & ERI & MV & EW & S\&P 500 \\
\hline CR & $35.87 \%$ & $31.00 \%$ & $22.28 \%$ & $-19.38 \%$ \\
\hline AR & $7.93 \%$ & $6.96 \%$ & $5.14 \%$ & $5.22 \%$ \\
\hline AS & 0.5448 & 0.3711 & 0.3170 & -0.0662 \\
\hline AST & 0.2306 & 0.1517 & 0.1301 & -0.0187 \\
\hline MD & $45.56 \%$ & $57.70 \%$ & $63.89 \%$ & $56.34 \%$ \\
\hline AC & 7.3987 & 1.00 & 243 & N/A \\
\hline AT & 0.0249 & 0.0000 & 0.01 & N /A \\
\hline PCA & $32.78 \%$ & $100.00 \%$ & $40.24 \%$ & N/A \\
\hline \hline
\end{tabular}

Table 4.3 Backtest statistics. Stocks with

Figure 4.4 Portfolio optimization back$\alpha \in(2.2,2.6)$

test. Stocks with $\alpha \in(2.2,2.6)$

If the stock selection is restricted to those with $\alpha$ between 2.2 and 2.6, the annualized return of the ERI based portfolio (7.93\%) is somewhat above the MV and EW benchmarks (6.96\% and 5.14\%, respectively). While the returns are on the same scale, the volatilities of the MV and the EW portfolios rare much higher. Thus ERI optimization clearly outperforms its peers in terms of Sharpe ratio, STARR (both higher for ERI), and maximal drawdown (lower for ERI). It is somewhat astonishing that the PCA of the MV portfolio is $100 \%$, i.e. the minimum variance algorithm selects only one stock.

\section{Selection from the set of stocks with $\alpha \geq 2.6$}

For stocks with $\alpha>2.6$ (and hence lightest tails), the performance of the ERI minimization strategy stays behind MV and EW in terms of annualized return, Sharpe ratio, STARR, and turnover. The maximal drawdown is similar for ERI and MV, and higher for the EW portfolio. The diversification level in terms of PCA is similar for all three competing strategies. The portfolio concentrations resulting from the ERI and the MV approaches are on the same level, and slightly higher for the ERI optimal portfolio.

Thus the impressive advantage of the ERI minimization strategy seems to be restricted to stocks with pronounced heavy-tail behaviour. This advantage turns into near parity for stocks with moderately heavy tails. For light-tailed stocks the MV strategy yields higher annualized returns with a similar drawdown, and the EW portfolio even higher returns, but also a significantly 
The final publications is now available at doi:10.1016/j.jempfin.2015.03.003

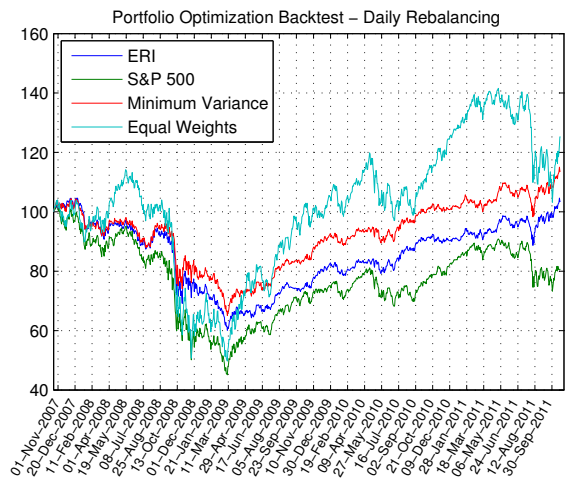

Figure 4.5 Portfolio optimization backtest. Stocks with $\alpha \geq 2.6$

\begin{tabular}{lcccc}
\hline \hline & ERI & MV & EW & S\&P 500 \\
\hline \hline CR & $3.47 \%$ & $13.62 \%$ & $25.27 \%$ & $-19.38 \%$ \\
\hline AR & $0.85 \%$ & $3.23 \%$ & $5.77 \%$ & $-5.22 \%$ \\
\hline AS & 0.1397 & 0.2636 & 0.3367 & -0.0462 \\
\hline AST & 0.0581 & 0.1114 & 0.1382 & -0.0187 \\
\hline MD & $42.58 \%$ & $43.43 \%$ & $64.89 \%$ & $56.34 \%$ \\
\hline AC & 3.4817 & 4.4715 & 67 & N/A \\
\hline AT & 0.0165 & 0.0091 & 0.01 & N/A \\
\hline PCA & $54.92 \%$ & $52.43 \%$ & $47.52 \%$ & N/A \\
\hline \hline
\end{tabular}

Table 4.4 Backtest statistics. $\alpha \geq 2.6$

higher maximal drawdown. These findings perfectly accord with model assumptions underlying these two methodologies: MV uses covariances, and ERI minimization is particularly applicable in cases when covariances do not exist or cannot be estimated reliably. On the other hand, ERI minimization strongly relies on the estimation of the tail index $\alpha$, which is known to become increasingly difficult for lighter tails - see, e.g., Embrechts et al. [15].

\subsection{Backtesting with an alternative estimator for $\alpha$}

To assess the suitability of the estimator we used for $\alpha$, we repeated our backtesting experiments with another estimation approach. The Hill estimator in (3.1) uses the tail fraction size $k$ as a parameter. The foregoing results are based on a static $10 \%$ rule, i.e. $k=150$. It is well known that the choice of the tail fraction size $k$ can have a strong influence on the resulting estimates - see, e.g., Embrechts et al. [15]. Thus, as an alternative to the static 10\% rule, we tried the recent adaptive approach by Nguyen and Samorodnitsky [37, which involves sequential statistical testing for polynomial tails. The results of this backtesting study are outlined below.

\section{Optimization over the entire set of stocks}

Figure 4.6 and Table 4.5 represent the basic setting without grouping the stocks according to the estimated tail index $\alpha$. It is a bit surprising that the adaptive choice of the tail fraction size $k$ does not improve the performance of the ERI based strategy. The annualized return is significantly lower than 
The final publications is now available at doi:10.1016/j.jempfin.2015.03.003

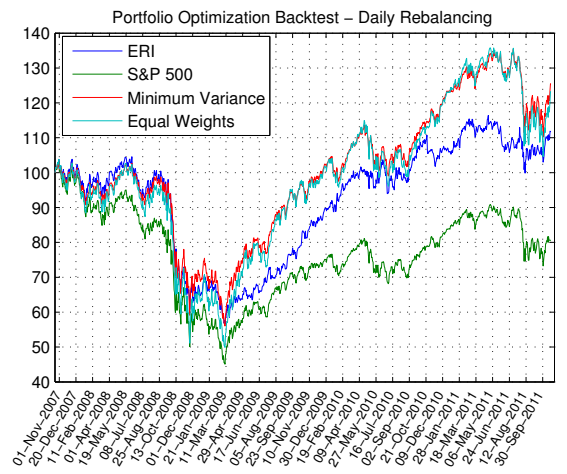

Figure 4.6 Alternative estimator $\hat{\alpha}$ : portfolio optimization backtest in the basic set-up (on all S\&P 500 stocks)

\begin{tabular}{lcccc}
\hline \hline & ERI & MV & EW & S\&P 500 \\
\hline \hline CR & $11.76 \%$ & $25.48 \%$ & $23.24 \%$ & $-19.38 \%$ \\
\hline AR & $2.81 \%$ & $5.81 \%$ & $5.34 \%$ & $-5.22 \%$ \\
\hline AS & 0.2360 & 0.3469 & 0.3229 & -0.0462 \\
\hline AST & 0.0939 & 0.1410 & 0.1318 & -0.0187 \\
\hline MD & $51.39 \%$ & $58.61 \%$ & $63.27 \%$ & $56.34 \%$ \\
\hline AC & 64.33 & 127.22 & 444 & N/A \\
\hline AT & 0.0381 & 0.0272 & 0.01 & N/A \\
\hline PCA & $46.48 \%$ & $35.49 \%$ & $38.80 \%$ & N/A \\
\hline \hline
\end{tabular}

Table 4.5 Alternative $\hat{\alpha}$ : backtest statistics in the basic set-up.

with the static $10 \%$ rule. The overall result clearly stays behind the MV and the EW benchmarks. The only aspect where ERI is still better is the maximal drawdown, but it cannot compensate for the lower overall return. The reason for this outcome is the lower value of the tail fraction size $k$ that is selected by the adaptive approach. Typical values are about 25 , and all values are lower than 150 that come from the static $10 \%$ rule. Thus the adaptive approach looks too far into the tail, where the scaling of excess probabilities may already be different from the scaling in the application range.

\section{Grouping the stocks according to the estimated $\alpha$}

As next step, we grouped the stocks according to their estimates. On average, the Nguyen-Samorodnitsky estimator gave higher values of $\alpha$, i.e., it indicated lighter tails than the static $10 \%$ rule. Therefore we chose a different grouping of the $\alpha$ values: $\alpha \leq 2.7, \alpha \in(2.7,4.5)$, and $\alpha \geq 4.5$. The backtesting results are presented in Table 4.6 .

In all three cases the annualized return of the ERI strategy is lower than that of the MV portfolio. Interestingly, the worst performance of the ERI based strategy occurs in the middle group, and not in the group with lightest tails. Possible explanations here may be the different composition of the three groups (heavy, moderate, or light tails) and also the different values of $\alpha$ used in the portfolio optimization algorithm.

All in all we can conclude that adaptive (and thus fully automatized) 
The final publications is now available at doi:10.1016/j.jempfin.2015.03.003

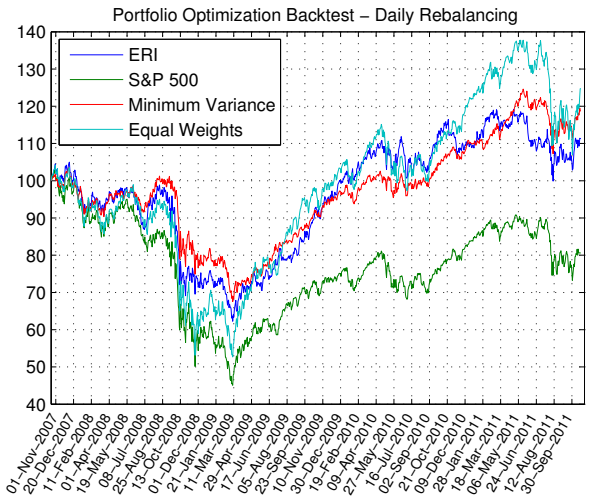

\begin{tabular}{lcccc}
\hline \hline & ERI & MV & EW & S\&P 500 \\
\hline \hline CR & $11.33 \%$ & $18.75 \%$ & $24.76 \%$ & $-19.38 \%$ \\
\hline AR & $2.71 \%$ & $4.37 \%$ & $5.66 \%$ & $-5.22 \%$ \\
\hline AS & 0.2317 & 0.3260 & 0.3354 & -0.0462 \\
\hline AST & 0.0958 & 0.1351 & 0.1362 & -0.0187 \\
\hline MD & $47.76 \%$ & $45.79 \%$ & $61.77 \%$ & $56.34 \%$ \\
\hline AC & 9.02 & 10.09 & 107 & N/A \\
\hline AT & 0.0385 & 0.0148 & 0.01 & N/A \\
\hline PCA & $43.65 \%$ & $30.87 \%$ & $33.46 \%$ & N/A \\
\hline \hline
\end{tabular}

(a) $\alpha \leq 2.7$

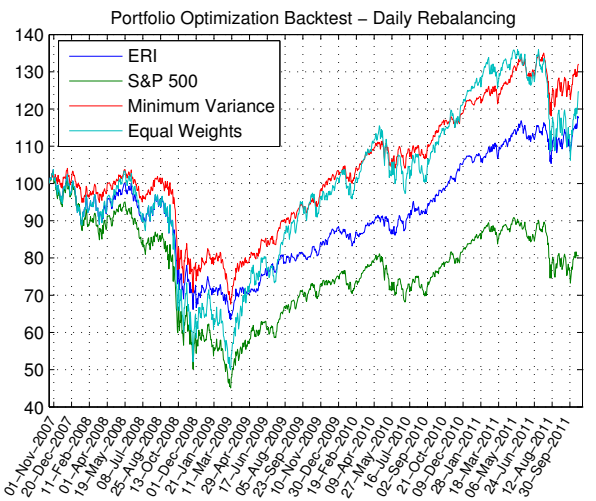

\begin{tabular}{lcccc}
\hline \hline & ERI & MV & EW & S\&P 500 \\
\hline \hline CR & $17.67 \%$ & $31.97 \%$ & $24.67 \%$ & $-19.38 \%$ \\
\hline AR & $4.14 \%$ & $7.15 \%$ & $5.64 \%$ & $-5.22 \%$ \\
\hline AS & 0.3015 & 0.4578 & 0.3318 & -0.0462 \\
\hline AST & 0.1202 & 0.1852 & 0.1365 & -0.0187 \\
\hline MD & $46.26 \%$ & $49.93 \%$ & $63.26 \%$ & $56.34 \%$ \\
\hline AC & 48.16 & 28.09 & 252 & N/A \\
\hline AT & 0.0384 & 0.0201 & 0.01 & N/A \\
\hline PCA & $48.29 \%$ & $32.56 \%$ & $40.25 \%$ & N/A \\
\hline \hline
\end{tabular}

(b) $\alpha \in(2.7,4.5)$

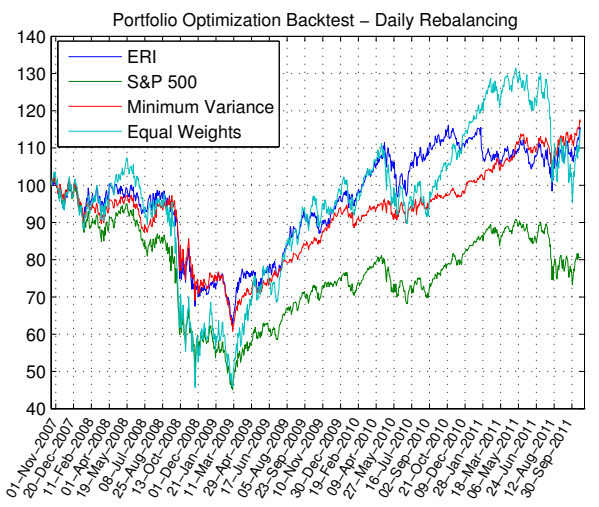

\begin{tabular}{lcccc}
\hline \hline & ERI & MV & EW & S\&P 500 \\
\hline \hline CR & $15.23 \%$ & $17.02 \%$ & $15.12 \%$ & $-19.38 \%$ \\
\hline AR & $3.59 \%$ & $3.99 \%$ & $3.57 \%$ & $-5.22 \%$ \\
\hline AS & 0.2699 & 0.2991 & 0.2810 & -0.0462 \\
\hline AST & 0.1155 & 0.1233 & 0.1143 & -0.0187 \\
\hline MD & $46.43 \%$ & $48.29 \%$ & $65.23 \%$ & $56.34 \%$ \\
\hline AC & 6.73 & 3.98 & 85 & N/A \\
\hline AT & 0.0326 & 0.0113 & 0.02 & N/A \\
\hline PCA & $58.04 \%$ & $45.34 \%$ & $44.54 \%$ & N/A \\
\hline \hline
\end{tabular}

(c) $\alpha \geq 4.5$

Table 4.6 Alternative $\hat{\alpha}$ : backtest statistics on stocks

23

(C)2015, Elsevier. Licensed under the Creative Commons Attribution-NonCommercial-NoDerivatives 4.0 International http: //creativecommons.org/licenses/by-nc-nd/4.0/ 
The final publications is now available at doi:10.1016/j.jempfin.2015.03.003

choice of the tail fraction size $k$ can be problematic in real applications. This can be explained by the tail orientation of the Nguyen-Samorodnitsky approach. Roughly speaking, it tests for polynomial tails and chooses the largest value of $k$ for which the test is still positive. While this is perfectly reasonable for data from an exact MRV model, there are at least two reasons why this method can fail on real data. First, if the data fails to satisfy the MRV assumption far out in the tail, the subsequent testing for small values of $k$ can be misleading. The second reason was already discussed in Section 2.2. If the polynomial scaling changes for different severities, then the scaling behaviour of the distribution in the application area can differ from what is suggested by the true, but too asymptotic tail index. Our backtesting results show that these issues are highly relevant in practice.

\subsection{Behaviour of portfolio characteristics over time}

We conclude our analysis by a comparison of the ways the competing portfolios behave over time. This allows for deeper insight and allows to discover some more points of difference.

\section{Concentration and portfolio composition}

We start with the development of the concentration coefficient (CC) introduced in (4.1). Its behaviour over time in the basic set-up (no grouping of stocks according to $\alpha$ ) is shown in Figure 4.7. This graphic shows that the number of stocks in the MV portfolio is permanently about 10 times higher than in the ERI optimal portfolio. The CC oscillation pattern suggests that the ERI based portfolio is more volatile in the crisis and much less volatile in benign periods.

This impression is confirmed by Figure 4.8. The dynamics of the MV Portfolio in Figure 4.9 is similar, but the difference between the crisis and recovery period is somewhat weaker. All in all it seems that the minimum variance portfolio undergoes many small changes, whereas the changes in the ERI optimal portfolio are less but much stronger.

\section{Turnover}

The impression about stronger changes in the ERI portfolio accords with the findings on the average portfolio turnover in Sections 4.1 and 4.2 . The 
The final publications is now available at doi:10.1016/j.jempfin.2015.03.003

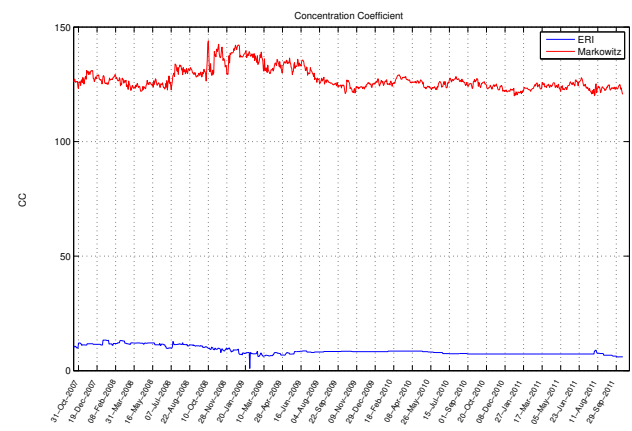

Figure 4.7 Concentration Coefficient in the backtesting experiment on all S\&P 500 stocks. Total set of stocks with $10 \%$ threshold alpha estimation.

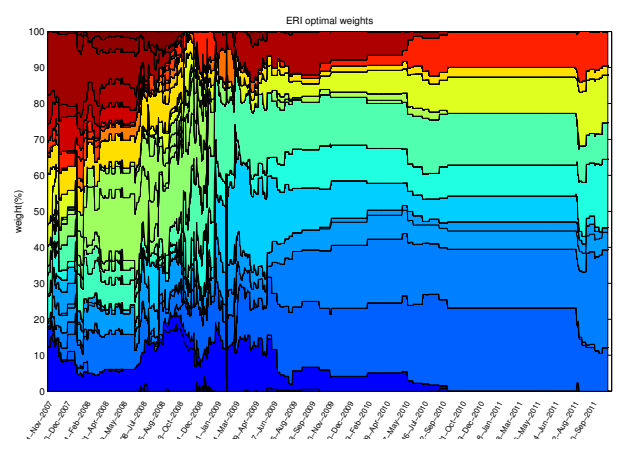

Figure 4.8 ERI optimal weights in backtesting on all S\&P 500 stocks

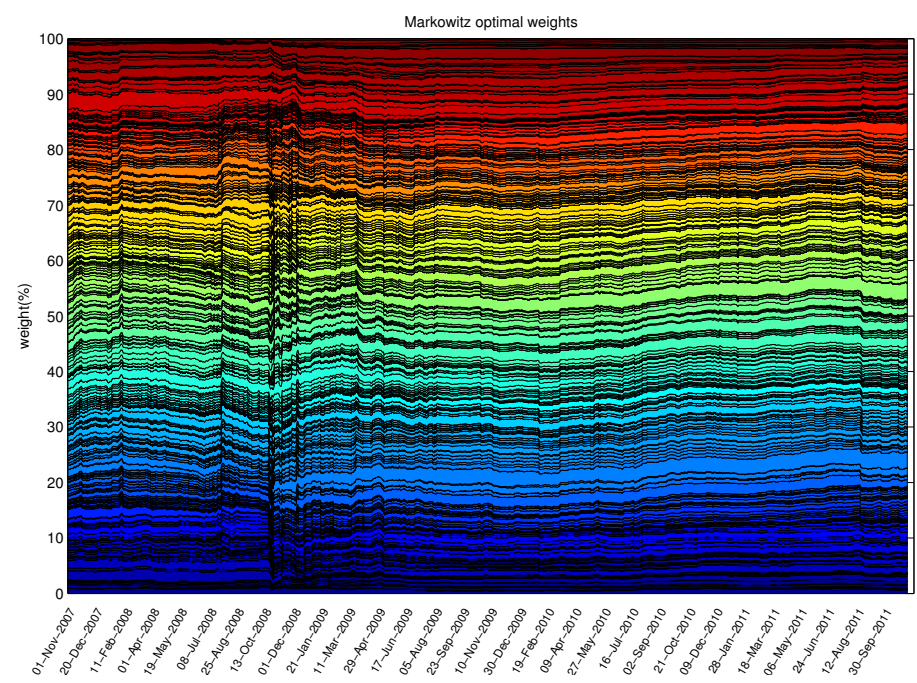

Figure 4.9 Weights of the minimum variance portfolio in backtesting on all S\&P 500 stocks

development of the turnover coefficient over time is shown in Figure 4.10, The larger the spikes in the turnover pattern, the greater the instantaneous portfolio shift. The difference between the ERI minimization and the MV portfolio in the crisis period is remarkable. The turnover pattern of the MV portfolio points to a lot of small portfolio changes that lead to permanent, but moderate trading activity. The pattern of the ERI based portfolio has a lower level of basic activity, but much greater spikes corresponding to large 
The final publications is now available at doi:10.1016/j.jempfin.2015.03.003

portfolio shifts. Thus, if carried out immediately, the restructuring of the ERI optimal portfolio requires more liquidity in the market. This disadvantageous feature can be tempered by splitting the transactions and distributing them over time. The tradeoff between fast reaction to events in the market and liquidity constraints is an interesting topic for further research.

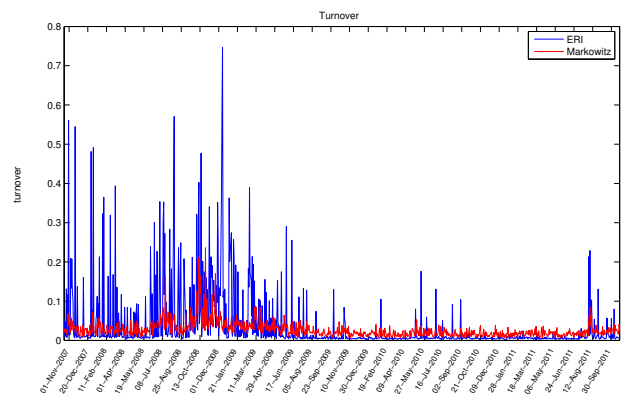

Figure 4.10 Portfolio turnover

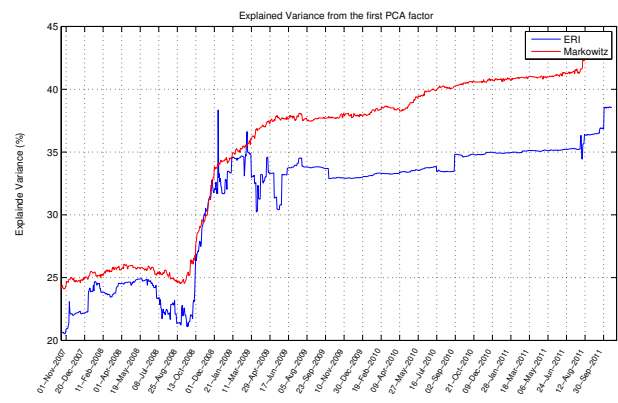

Figure 4.11 Variance explained by the first PCA factor

\section{Diversification measured by PCA}

The development of the first PCA factor over time is shown in Figure 4.11. The amount of portfolio variance that can be explained by the first PCA factor increases in the months after the default of Lehman Brothers to a new level. This shows that the recent financial crisis has changed the perception of dependence in the market and thus increased the dependence between the stocks. Ranging below $25 \%$ before the crisis, the first PCA factors of both strategies are typically above $35 \%$ afterwards. This chart indicates a change in the intrinsic market dynamics. The stronger co-movements of S\&P 500 stocks reflect the new perception of systemic risk. As a consequence, the diversification potential in the after-crisis period is lower than in the time before the crisis.

Most of the time, the first PCA factor of the ERI optimal portfolio ranges somewhat below that of the Markowitz portfolio. Thus we can conclude that ERI optimization brings more diversity into the portfolio than the mean-variance approach. To the use of PCA: There is one exception to this rule: in February 2009, the first PCA factor of the ERI optimal portfolio peaks out to $100 \%$. It corresponds to a single day when the ERI strategy selects only one stock for the investment portfolio. On this remarkable day, the first PCA 
The final publications is now available at doi:10.1016/j.jempfin.2015.03.003

factor is obviously identical with the investment portfolio. Recalculations let to slightly different weights but to almost identical portfolio returns. Results of this kind can be avoided in practice by appropriate bounds on portfolio restructuring.

\section{Tail index estimates}

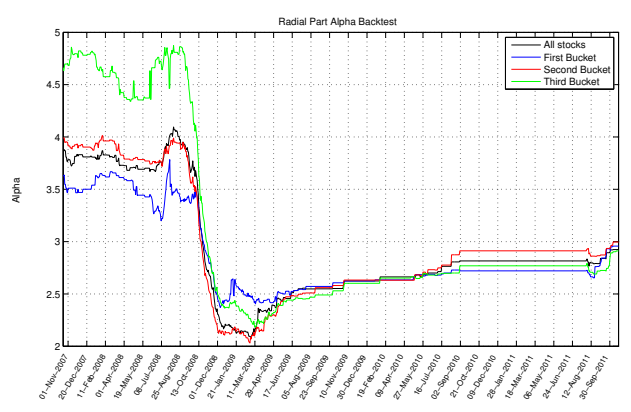

Figure 4.12 Estimated tail index $\alpha$ for the radial part of S\&P 500 stocks and three subgroups

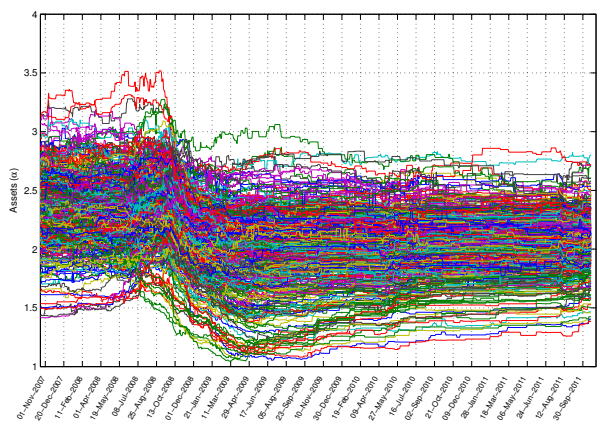

Figure 4.13 Estimated tail index $\alpha$ for the S\&P 500 stocks

In addition to the backtesting studies where the tail index $\alpha$ is estimated for the radial part of the random vector $X$, we also estimated $\alpha$ for each stock separately. The estimated values of $\alpha$ for the radial parts are shown in Figure 4.12, and the development of $\alpha$ estimates for single stocks is shown in Figure 4.13. Beginning in summer 2008, there is a common downside trend for all stocks, i.e. all return tails become heavier in the crisis time. This trend stops in spring 2009. The missing recovery since then can be explained by the width of the estimation window. Based on the foregoing 1500 trading days, our estimators remain influenced by the crisis for 6 years. This effect is visible in both figures. In addition to that, Figure 4.12 shows that after the crisis the estimated values of $\alpha$ in all three sub-groups are very close to each other and even change their ordering compared to the pre-crisis period: the group with lowest $\alpha$ before crisis does not give the lowest $\alpha$ after the crisis. These effects may be explained by the strong influence of extremal events during the crisis on the estimates in the after-crisis period. As the historical observation window includes $n=1500$ days, the crisis events do not disappear from this window until the end of the backtesting period. It seems that the estimated values of $\alpha$ tend to ignore the recovery of the stocks 
The final publications is now available at doi:10.1016/j.jempfin.2015.03.003

in the after-crisis period. This may be one more explanation to the different performance of the ERI strategy in the different stock groups. This effect can be tempered by downweighting the observations in the historical window when they move away from the present time. The choice of this weighting rule goes beyond the scope of this paper and should be studied separately.

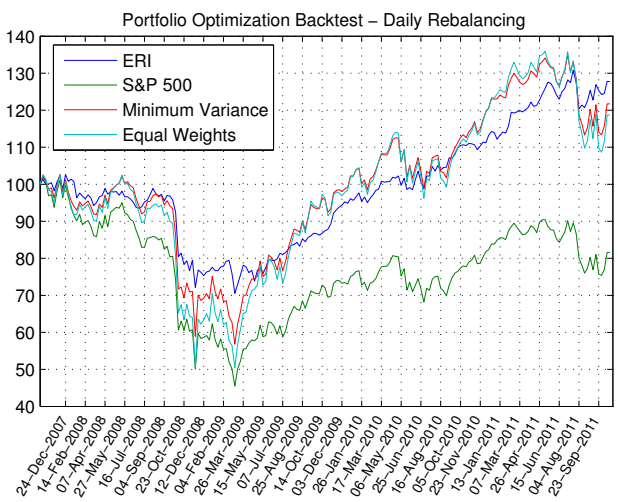

\begin{tabular}{lcccc}
\hline \hline & ERI & MV & EW & S\&P 500 \\
\hline CR & $27.77 \%$ & $21.73 \%$ & $18.69 \%$ & $-9.38 \%$ \\
\hline $\mathrm{AR}$ & $6.31 \%$ & $5.03 \%$ & $4.37 \%$ & $-5.22 \%$ \\
\hline $\mathrm{AS}$ & 0.4864 & 0.3216 & 0.2949 & -0.0462 \\
\hline $\mathrm{AST}$ & 0.1756 & 0.1307 & 0.1236 & -0.087 \\
\hline $\mathrm{MD}$ & $46.08 \%$ & $57.92 \%$ & $62.85 \%$ & $56.34 \%$ \\
\hline $\mathrm{AC}$ & 8.71 & 127.29 & 444 & $\mathrm{~N} / \mathrm{A}$ \\
\hline $\mathrm{AT}$ & 0.0471 & 0.0406 & 0.03 & $\mathrm{~N} / \mathrm{A}$ \\
\hline $\mathrm{PCA}$ & $31.19 \%$ & $35.40 \%$ & $38.71 \%$ & $\mathrm{~N} / \mathrm{A}$ \\
\hline
\end{tabular}

(a) all stocks

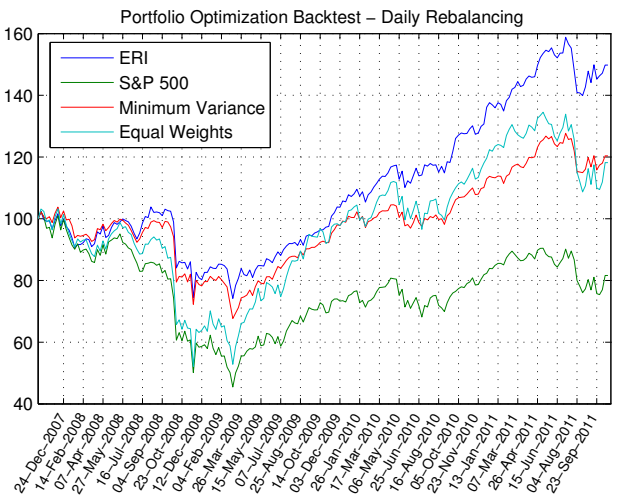

\begin{tabular}{lcccc}
\hline \hline & ERI & MV & EW & S\&P 500 \\
\hline CR & $49.78 \%$ & $20.41 \%$ & $18.20 \%$ & $-19.38 \%$ \\
\hline AR & $10.61 \%$ & $4.74 \%$ & $4.26 \%$ & $-5.22 \%$ \\
\hline AS & 0.6491 & 0.3581 & 0.2904 & -0.0462 \\
\hline AST & 0.2518 & 0.1318 & 0.1205 & -0.0187 \\
\hline MD & $53.32 \%$ & $47.01 \%$ & $61.24 \%$ & $56.34 \%$ \\
\hline AC & 7.45 & 11.00 & 134 & N/A \\
\hline AT & 0.0324 & 0.0231 & 0.03 & N/A \\
\hline PCA & $34.94 \%$ & $33.23 \%$ & $35.08 \%$ & N /A
\end{tabular}

(b) $\alpha \leq 2.2$

Table 4.7 Weekly rebalancing: backtest statistics

Another issue that may be relevant here is the sensitivity of tail estimators (including Hill's $\widehat{\alpha}$ ) to non-i.i.d. data and volatility clustering. Consistency and asymptotic normality results for tail estimators require that $n \rightarrow \infty$, $k \rightarrow \infty$ and $k / n \rightarrow 0$ where $k=k(n)$ is the number of observations considered extremal (we use $n=1500$ and $k=150$ ). Asymptotically, volatility clustering featured in many popular models (e.g. GARCH) increases the effective sample size by the reciprocal value of the average cluster size. In 
The final publications is now available at doi:10.1016/j.jempfin.2015.03.003

addition to these asymptotic results, finite sample behaviour of each particular estimator can be relevant as well (cf. Chavez-Demoulin and Davison [4], Drees [13], and references therein).

\subsection{Weekly rebalancing}

In this part of the study we switch from daily to weekly rebalancing. The calculation of portfolio weights is still based on daily data. This allows to use all observations in the historical window, and not only the weekly returns. In some sense, trading once a week reflects the delayed execution of large orders. To avoid moving the market, trading of high volumes is often split into parts and executed step by step.

The results of this experiment are shown in Tables 4.7 and 4.8 . For simplicity, the numbers for S\&P 500 are taken from the tables on daily rebalancing. The overall picture is very similar to the daily rebalancing set-up: ERI compares favourable to its peers in all backtesting runs except for the one experiment with light-tail stocks. The particularly high performance improvement on stocks with heavy tails achieved with daily rebalancing can also be achieved with weekly rebalancing.

\section{Conclusions}

Our backtesting results suggest that the Extreme Risk Index (ERI) could be useful in practice. Comparing basic implementations of the ERI methodology with the minimum-variance (MV) portfolio and the equally weighted (EW) portfolio, we obtained promising results for stocks with heavy tails. Tailored to such assets, the ERI optimal portfolio not only outperforms MV and EW portfolios, but it also yields an annualized return of $11.5 \%$ over 4 years including the financial crisis of 2008. This advantage should outweigh the higher transaction costs caused by the ERI based approach. Thus, taking into account the special nature of diversification for heavy-tailed asset returns, the ERI strategy increases the reward for the corresponding risks.

Our study also shows that the MV and EW approaches can catch up with ERI optimization in some cases, especially when applied to stocks with lighter tails. Therefore a combined algorithm switching between ERI and variance as risk measure (depending on the current volatility in the market) may be a good choice. First empirical studies confirm this. However, the results

(C)2015, Elsevier. Licensed under the Creative Commons Attribution-NonCommercial-NoDerivatives 4.0 International

http: //creativecommons.org/licenses/by-nc-nd/4.0/ 
The final publications is now available at doi:10.1016/j.jempfin.2015.03.003

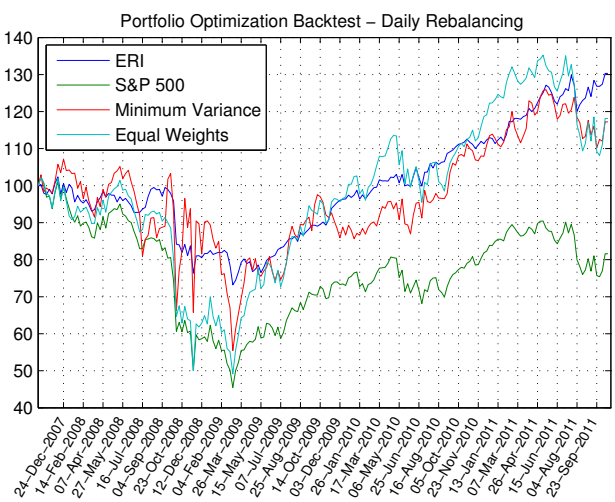

\begin{tabular}{lcccc}
\hline \hline & ERI & MV & EW & S\&P 500 \\
\hline CR & $30.31 \%$ & $17.23 \%$ & $18.13 \%$ & $-19.38 \%$ \\
\hline AR & $6.83 \%$ & $4.05 \%$ & $4.25 \%$ & $-5.22 \%$ \\
\hline AS & 0.5348 & 0.3064 & 0.2921 & -0.04620 \\
\hline AST & 0.1935 & 0.1273 & 0.1232 & -0.0187 \\
\hline MD & $43.82 \%$ & $55.95 \%$ & $63.68 \%$ & $56.34 \%$ \\
\hline AC & 7.41 & 1.00 & 243 & N/A \\
\hline AT & 0.0337 & 0.0000 & 0.03 & N/A \\
\hline PCA & $32.74 \%$ & $100.00 \%$ & $40.16 \%$ & N/A
\end{tabular}

(a) $\alpha \in(2.2,2.6)$

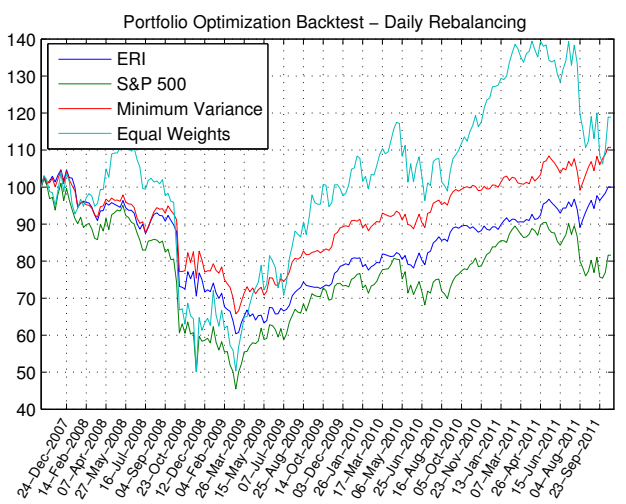

\begin{tabular}{lcccc}
\hline \hline & ERI & MV & EW & S\&P 500 \\
\hline CR & $0.04 \%$ & $10.72 \%$ & $18.90 \%$ & $-19.38 \%$ \\
\hline AR & $0.01 \%$ & $2.57 \%$ & $4.41 \%$ & $-5.22 \%$ \\
\hline AS & 0.0884 & 0.2378 & 0.3012 & 0.0000 \\
\hline AST & 0.0343 & 0.0957 & 0.1281 & 0.0000 \\
\hline MD & $42.33 \%$ & $40.60 \%$ & $64.01 \%$ & $0.00 \%$ \\
\hline AC & 3.49 & 4.48 & 67 & N/A \\
\hline AT & 0.0189 & 0.0162 & 0.03 & N/A \\
\hline PCA & $54.81 \%$ & $52.27 \%$ & $47.44 \%$ & N/A
\end{tabular}

(b) $\alpha \geq 2.6$

Table 4.8 Weekly rebalancing: backtest statistics

obtained so far are not very stable, and the choice of the switching strategy needs a deeper investigation. Other improvements of the ERI methodology may be achieved by downweighting the crisis events when they reach the far end of the historical observation window and by smoothing the pattern of trading activities. All these questions will be subject of further research.

\section{Acknowledgement}

The authors thank Svetlozar T. Rachev who suggested to undertake a backtesting study of the ERI methodology and for his help to organize this study.

The authors are grateful to a reviewer for several thoughtful suggestions 
The final publications is now available at doi:10.1016/j.jempfin.2015.03.003

and hints to relevant literature which lead to a strong improvement of our paper.

\section{References}

\section{References}

[1] A. Araujo and E. Giné. The Central Limit Theorem for Real and Banach Valued Random Variables. Wiley, 1980.

[2] C. B. Barry. Portfolio analysis under uncertain means, variances, and covariances. The Journal of Finance, 29(2):515-522, 1974. doi:10.1111/j.1540-6261.1974.tb03064.x.

[3] F. Black and R. Litterman. Asset allocation: combining investor views with market equilibrium. The Journal of Fixed Income, 1(2):7-18, 1991. doi:10.3905/jfi.1991.408013

[4] V. Chavez-Demoulin and A. C. Davison. Modelling time series extremes. REVSTAT - Statistical Journal, 10(1):109-133, 2012. URL https:// www.ine.pt/revstat/pdf/rs120105.pdf.

[5] L. Chollete, V. de la Peña, and C.-C. Lu. International diversification: An extreme value approach. Journal of Banking \& Finance, 36(3):871885, 2012. doi:http://dx.doi.org/10.1016/j.jbankfin.2011.09.015.

[6] V. K. Chopra and W. T. Ziemba. The effect of errors in means, variances, and covariances on optimal portfolio choice. The Journal of Portfolio Management, 19(2):6-11, 1993. doi:10.3905/jpm.1993.409440.

[7] J. Daníelsson, L. de Haan, L. Peng, and C. G. de Vries. Using a bootstrap method to choose the sample fraction in tail index estimation. Journal of Multivariate Analysis, 76(2):226-248, 2001. doi:10.1006/jmva.2000.1903.

[8] V. DeMiguel and F. J. Nogales. Portfolio selection with robust estimation. Operations Research, 57(3):560-577, 2009. doi:10.1287/opre.1080.0566.

31

(C)2015, Elsevier. Licensed under the Creative Commons Attribution-NonCommercial-NoDerivatives 4.0 International http: // creativecommons. org/licenses/by-nc-nd/4.0/ 
The final publications is now available at doi:10.1016/j.jempfin.2015.03.003

[9] V. DeMiguel, L. Garlappi, and R. Uppal. Optimal versus naive diversification: How inefficient is the $1 / \mathrm{n}$ portfolio strategy? Review of Financial Studies, 22(5):1915-1953, 2009. doi:10.1093/rfs/hhm075.

[10] F. Desmoulins-Lebeault and C. Kharoubi-Rakotomalalaé. Non-gaussian diversification: When size matters. Journal of Banking \&3 Finance, 36 (7):1987-1996, 2012. doi:10.1016/j.jbankfin.2012.03.006.

[11] F. J. DiTraglia and J. R. Gerlach. Portfolio selection: An extreme value approach. Journal of Banking \&3 Finance, 37(2):305-323, 2013. doi:10.1016/j.jbankfin.2012.08.022.

[12] T. Doganoglu, C. Hartz, and S. Mittnik. Portfolio optimization when risk factors are conditionally varying and heavy tailed. Computational Economics, 29(3-4):333-354, 2007. doi:10.1007/s10614-006-9071-1.

[13] H. Drees. Extreme quantile estimation for dependent data, with applications to finance. Bernoulli, 9(1):617-657, 2003. doi: $10.3150 / \mathrm{bj} / 1066223272$.

[14] H. Drees and E. Kaufmann. Selecting the optimal sample fraction in univariate extreme value estimation. Stochastic Processes and their Applications, 75(2):149-172, 1998. doi:10.1016/S0304-4149(98)00017-9.

[15] P. Embrechts, C. Klüppelberg, and T. Mikosch. Modelling Extremal Events. Springer, 1997.

[16] J. Fletcher and S. Leyffer. Solving mixed integer nonlinear programs by outer approximation. Math. Programming, 66:327-349, 1994. doi:10.1007/BF01581153

[17] L. Garlappi, R. Uppal, and T. Wang. Portfolio selection with parameter and method uncertainty: A multi-prior approach. The Review of Financial Studies, 20:41-82, 2007. doi:10.1093/rfs/hhl003.

[18] L. E. Ghaoui, M. Oks, and F. Oustry. Worst-case value-at-risk and robust portfolio optimization: A conic programming approach. Operations Research, 51(4):543-556, 2003. doi:10.1287/opre.51.4.543.16101.

[19] D. Goldfarb and G. Iyengar. Robust portfolio selection problems. Mathematics of Operations Research, 28(1):1-38, 2003. doi:10.1287/moor.28.1.1.14260.

32

(C)2015, Elsevier. Licensed under the Creative Commons Attribution-NonCommercial-NoDerivatives 4.0 International http: //creativecommons.org/licenses/by-nc-nd/4.0/ 
The final publications is now available at doi:10.1016/j.jempfin.2015.03.003

[20] R. R. Grauer and N. H. Hakansson. Stein and $\{\mathrm{CAPM}\}$ estimators of the means in asset allocation. International Review of Financial Analysis, 4(1):35-66, 1995. doi:10.1016/1057-5219(95)90005-5.

[21] X. D. He and X. Y. Zhou. Portfolio choice via quantiles. Mathematical Finance, 21(2):203-231, 2011. doi:10.1111/j.1467-9965.2010.00432.x.

[22] U. Herold and R. Maurer. Portfolio choice and estimation risk. A comparison of bayesian to heuristic approaches. Astin Bulletin, 36 (1):135-160, 2006. URL http://journals.cambridge.org/article_ S0515036100014434.

[23] B. M. Hill. A simple general approach to inference about the tail of a distribution. The Annals of Statistics, 3(5):1163-1174, 1975. doi:10.1214/aos/1176343247.

[24] W. Hu and A. N. Kercheval. Portfolio optimization for student $\mathrm{t}$ and skewed t returns. Quantitative Finance, 10(1):91-105, 2010. doi:10.1080/14697680902814225.

[25] H. Hult and F. Lindskog. Multivariate extremes, aggregation and dependence in elliptical distributions. Advances in Applied Probability, 34 (3):587-608, 2002. doi:10.1239/aap/1033662167.

[26] N. Hyung and C. G. de Vries. Portfolio selection with heavy tails. Journal of Empirical Finance, 14(3):383-400, 2007. doi:10.1016/j.jempfin.2006.06.004.

[27] R. Jagannathan and T. Ma. Risk reduction in large portfolios: Why imposing the wrong constraints helps. Journal of Finance, 58:16511683, 2003. doi:10.1111/1540-6261.00580.

[28] R. Jarrow and F. Zhao. Downside loss aversion and portfolio management. Management Science, 52(4):558-566, 2006. doi: $10.1287 / \mathrm{mnsc} .1050 .0486$.

[29] P. Jorion. International portfolio diversification with estimation risk. The Journal of Business, 58(3):259-278, 1985. URL http://www. jstor.org/stable/2352997.

33

(C)2015, Elsevier. Licensed under the Creative Commons Attribution-NonCommercial-NoDerivatives 4.0 International http: //creativecommons.org/licenses/by-nc-nd/4.0/ 
The final publications is now available at doi:10.1016/j.jempfin.2015.03.003

[30] P. Jorion. Bayes-stein estimation for portfolio analysis. Journal of Financial and Quantitative Analysis, 21:279-292, 1986. doi: $10.2307 / 2331042$.

[31] P. Jorion. Bayesian and CAPM estimators of the means: Implications for portfolio selection. Journal of Banking \& Finance, 15(3):717-727, 1991. doi:10.1016/0378-4266(91)90094-3.

[32] G. Mainik. On Asymptotic Diversification Effects for Heavy-Tailed Risks. PhD thesis, University of Freiburg, 2010. URL http://www. freidok.uni-freiburg.de/volltexte/7510/pdf/thesis.pdf.

[33] G. Mainik. Estimating asymptotic dependence functionals in multivariate regularly varying models. Lithuanian Mathematical Journal, 52(3): 259-281, 2012. doi:10.1007/s10986-012-9172-6.

[34] G. Mainik and P. Embrechts. Diversification in heavy-tailed portfolios: properties and pitfalls. Annals of Actuarial Science, 7:26-45, 2013. doi:10.1017/S1748499512000280.

[35] G. Mainik and L. Rüschendorf. On optimal portfolio diversification with respect to extreme risks. Finance and Stochastics, 14:593-623, 2010. doi:10.1007/s00780-010-0122-z.

[36] H. Markowitz. Portfolio selection. The Journal of Finance, 7(1):77-91, 1952. URL http://www . jstor .org/stable/2975974.

[37] T. Nguyen and G. Samorodnitsky. Tail inference: where does the tail begin? Extremes, 15(4):437-461, 2012. doi:10.1007/s10687-011-0145-7.

[38] S. Ortobelli, S. T. Rachev, and F. J. Fabozzi. Risk management and dynamic portfolio selection with stable Paretian distributions. Journal of Empirical Finance, 17(2):195-211, 2010. doi:10.1016/j.jempfin.2009.09.002.

[39] S. T. Rachev, C. Menn, and F. J. Fabozzi. Fat-Tailed and Skewed Asset Return Distributions: Implications for Risk Management, Portfolio Selection, and Option Pricing. Wiley, 2005.

[40] S. I. Resnick. Heavy-Tail Phenomena. Springer, 2007.

34

(C)2015, Elsevier. Licensed under the Creative Commons Attribution-NonCommercial-NoDerivatives 4.0 International http: //creativecommons.org/licenses/by-nc-nd/4.0/ 
The final publications is now available at doi:10.1016/j.jempfin.2015.03.003

[41] S. I. Resnick and C. Stărică. Smoothing the Hill estimator. Advances in Applied Probability, 29(1):271-293, 1997. URL http://www.jstor. org/stable/1427870.

[42] R. Tütüncü and M. Koenig. Robust asset allocation. Annals of Operations Research, 132(1-4):157-187, 2004. doi:10.1023/B:ANOR.0000045281.41041.ed.

[43] M. R. Young. A minimax portfolio selection rule with linear programming solution. Management Science, 44(5):673-683, 1998. doi: $10.1287 /$ mnsc. 44.5 .673 .

[44] C. Zhou. Dependence structure of risk factors and diversification effects. Insurance: Mathematics and Economics, 46(3):531-540, 2010. doi:10.1016/j.insmatheco.2010.01.010

35

(C)2015, Elsevier. Licensed under the Creative Commons Attribution-NonCommercial-NoDerivatives 4.0 International http: //creativecommons.org/licenses/by-nc-nd/4.0/ 